\section{Vol. 31, Issue 5, May 2008}

Obituary

221 Steven Karger (1959-2008)

\section{Editorials}

222 Preoperative Staging of Rectal Tumors: What is the Most Optimal Staging Method?

Beets-Tan, R.G.H.; Beets, G.L. (Maastricht)

224 Small Molecule Inhibitors of Tyrosine Kinase: Waiting for the Good News in Colorectal Cancer as Well

Sobrero, A.; Andretta, V. (Genova)

226 When to Perform Surgery in Stage IV Melanoma Patients?

de Wilt, J.H.W.; Eggermont, A.M.M. (Rotterdam)

Original Articles

230 Preoperative Staging of Rectal Tumors: Comparison of Endorectal Ultrasound, Hydro-CT and HighResolution Endorectal MRI

Dinter, D.J.; Hofheinz, R.D.; Hartel, M.; Kaehler, G.F.A.B.; Neff, K.W.; Diehl, S.J. (Mannheim)

237 Gefitinib in Combination with Oxaliplatin and 5-Fluorouracil in Irinotecan-Refractory Patients with Colorectal Cancer: A Phase I Study of the Arbeitsgemeinschaft Internistische Onkologie (AIO) Hartmann, J.T.; Pintoffl, J.P. (Tübingen); Kröning, H. (Magdeburg); Bokemeyer, C. (Tübingen); Holtmann, M.; Höhler, T. (Mainz)

243 Increased CD4+CD25+FOXP3+ Regulatory T Cells in Cancer Patients from Conversion of CD4+CD25T Cells through Tumor-Derived Factors Mao, C.; Wang, S.; Jiang, Q.; Tong, J.; Ma, J.; Yang, M.; Xu, X.; Qiu, G.; Shao, Q.; Li, L.; Xu, H. (Zhenjiang)

251 Long-Term Outcome after Combined Radiochemotherapy for Anal Cancer - Retrospective Analysis of Efficacy, Prognostic Factors, and Toxicity Mai, S.K.; Welzel, G.; Hermann, B.; Bohrer, M.; Wenz, F. (Mannheim)
Band 31, Heft 5, Mai 2008

Obituary

221 Steven Karger (1959-2008)

Editorials

222 Präoperatives Staging von Rektaltumoren: Was ist die beste Methode?

Beets-Tan, R.G.H.; Beets, G.L. (Maastricht)

224 «Small Molecule»-Tyrosinkinaseinhibitoren: Wann kommen die guten Nachrichten auch für das Kolorektalkarzinom? Sobrero, A.; Andretta, V. (Genova)

226 Wann sollten Melanom-Patienten im Stadium IV operiert werden? de Wilt, J.H.W.; Eggermont, A.M.M. (Rotterdam)

Originalarbeiten

230 Präoperative Stadieneinteilung von Rektumkarzinomen: Vergleich von transrektalem Ultraschall, Hydro-CT und Kernspintomographie mit Endorektalspule Dinter, D.J.; Hofheinz, R.D.; Hartel, M.; Kaehler, G.F.A.B.; Neff, K.W.; Diehl, S.J. (Mannheim)

237 Gefitinib in Kombination mit Oxaliplatin und 5-Fluorouracil bei Irinotecan-refraktären Patienten mit kolorektalem Karzinom: Eine Phase-I-Studie der Arbeitsgemeinschaft Internistische Onkologie (AIO) Hartmann, J.T.; Pintoffl, J.P. (Tübingen); Kröning, H. (Magdeburg); Bokemeyer, C. (Tübingen); Holtmann, M.; Höhler, T. (Mainz)

243 Erhöhte Anzahl an CD4+CD25+FOXP3+ regulatorischen $\mathrm{T}-Z$ Zllen bei Krebspatienten durch Konversion von CD4+CD25- T-Zellen durch aus Tumorgewebe stammende Faktoren Mao, C.; Wang, S.; Jiang, Q.; Tong, J.; Ma, J.; Yang, M.; Xu, X.; Qiu, G.; Shao, Q.; Li, L.; Xu, H. (Zhenjiang)

251 Langzeitüberleben nach kombinierter Radiochemotherapie bei Analkarzinom - eine retrospektive Analyse von Effektivität, Prognostischen Faktoren und Toxizität Mai, S.K.; Welzel, G.; Hermann, B.; Bohrer, M.; Wenz, F. (Mannheim)

\section{KARGER}

Fax +497614520714

E-mail Information@Karger.de

www.karger.com
(C) 2008 S. Karger GmbH, Freiburg

Artikel (Volltext) und Inhaltsverzeichnisse

sowie das vorläufige Inhaltsverzeichnis des nächsten Heftes: www.karger.com/onk_bk.htm 


\section{Vol. 31, Issue 5, May 2008}

Clinical Cases

259 Long-Term Survival in Stage IV Melanoma after Repetitive Surgical Therapy Tomov, T.; Siegel, R.; Bembenek, A. (Berlin)

262 Perforation of the Superior Vena Cava - a Rare Complication of Central Venous Catheters

Jost, K.; Leithäuser, M.; Große-Thie, C.; Bartolomaeus, A.; Hilgendorf, I.; Andree, H.; Casper, J.; Freund, M.; Junghanss, C. (Rostock)

266 Male Breast Cancer 15 Years after Allogeneic Hematopoietic Cell Transplantation Including Total Body Irradiation for Recurrent Acute Lymphoblastic Leukemia

Lowe, T.; Luu, T.; Shen, J.; Bhatia, S.; Shibata, S.; Stein, A.; Somlo, G. (Duarte, CA)

\section{Review Article}

271 Mitomycin $\mathrm{C}$ in the Treatment of Gastrointestinal Tumours: Recent Data and Perspectives Hofheinz, R.D. (Mannheim); Beyer, U. (Wedel); Al-Batran, S.E. (Frankfurt); Hartmann, J.T. (Tübingen)

283 PharmaForum

288 PharmaNews / PharmaTicker

291 Meetings and Conferences

249 Imprint

Forthcoming papers are listed on page 292.

\section{Band 31, Heft 5, Mai 2008}

Kasuistiken

259 Langzeitüberleben einer Patientin mit malignem Melanom im Stadium IV nach multipler chirurgischer Therapie Tomov, T.; Siegel, R.; Bembenek, A. (Berlin)

262 Perforation der Vena cava superior - eine seltene Komplikation zentraler Venenkatheter Jost, K.; Leithäuser, M.; Große-Thie, C.; Bartolomaeus, A.; Hilgendorf, I.; Andree, H.; Casper, J.; Freund, M.; Junghanss, C. (Rostock)

266 Mammakarzinom beim Mann 15 Jahre nach allogener hämatopoetischer Zelltransplantation mit Ganzkörperbestrahlung wegen rezidivierender akuter lymphoblastischer Leukämie Lowe, T.; Luu, T.; Shen, J.; Bhatia, S.; Shibata, S.; Stein, A.; Somlo, G. (Duarte, CA)

\section{Übersichtsarbeit}

271 Mitomycin in der Behandlung gastrointestinaler Tumoren: Aktuelle Daten und Perspektiven Hofheinz, R.D. (Mannheim); Beyer, U. (Wedel); Al-Batran, S.E. (Frankfurt); Hartmann, J.T. (Tübingen)

283 PharmaForum

288 PharmaNews / PharmaTicker

291 Tagungen und Kongresse

249 Impressum

Einen Ausblick auf den Inhalt der kommenden Hefte finden Sie auf Seite 292.

\section{KARGER}

Fax +497614520714

E-mail Information@Karger.de

www.karger.com
(C) 2008 S. Karger GmbH, Freiburg 